\title{
Se-methylselenocysteine sensitized TRAIL-mediated apoptosis via down-regulation of Bcl-2 expression
}

\author{
JUNG TAE LEE ${ }^{1}$, TAE-JIN LEE ${ }^{2}$, JONG-WOOK PARK ${ }^{1}$ and TAEG KYU KWON ${ }^{1}$ \\ ${ }^{1}$ Department of Immunology and Chronic Disease Research Center and Institute for Medical Science, School of Medicine, \\ Keimyung University, 194 DongSan-Dong Jung-Gu, Taegu 700-712; ${ }^{2}$ Department of Anatomy, College of Medicine, \\ Yeungnam University, 317-1 Daemyoung-Dong Nam-Gu, Taegu 705-717, Korea
}

Received December 15, 2008; Accepted February 12, 2009

DOI: 10.3892/ijo_00000274

\begin{abstract}
Recent studies establish a critical role of selenium in cancer prevention in vitro and in vivo. Selenium may sensitize TRAIL-mediated apoptosis in human renal cancer cells and increase therapeutic efficacy. In this study, we demonstrate that concomitant administration of TRAIL and Se-methylselenocysteine (Se-MSC) produces synergistic effects on the induction of apoptosis in Caki cells. Se-MSC rapidly and specifically down-regulates expression of the Bcl-2 at transcriptional level. The forced expression of Bcl-2 attenuated Se-MSC plus TRAIL-mediated apoptosis, suggesting that the lessened $\mathrm{Bcl}-2$ expression caused by Se-MSC treatment is critical to the increased sensitivity to TRAIL in renal cancer cells. In addition, we demonstrate that the synergistic effects of Se-MSC and TRAIL result from the activation of the caspase-dependent pathways. Co-administration of HA14-1, a small molecule Bcl-2 inhibitor and TRAIL increased apoptosis in Caki cells. Taken together, Se-MSC-mediated down-regulation of Bcl-2 is able to sensitize Caki cells for TRAIL-induced apoptosis. Thus, seleniumbased dietary compounds may help to overcome resistance to TRAIL-mediated apoptosis in renal cancer cells.
\end{abstract}

\section{Introduction}

Tumor necrosis factor-related apoptosis-inducing ligand (TRAIL) induces apoptosis in a wide variety of transformed and cancer cells but not in non-neoplastic cells (1). Since TRAIL is a tumor-selective and apoptosis-inducing cytokine, it is considered to be a promising new candidate for cancer prevention and treatment. However, recent studies have shown that some cancer cells are resistant to the apoptotic effects of TRAIL (2-4). TRAIL-resistant cancer cells can be sensitized by chemotherapeutic drugs in vitro, indicating that combination

Correspondence to: Dr Taeg Kyu Kwon, Department of Immunology, School of Medicine, Keimyung University, 194 DongSanDong Jung-Gu, Taegu 700-712, Korea

E-mail: kwontk@dsmc.or.kr

Key words: Se-MSC, TRAIL, Bcl-2, HA14-1 therapy may be a possibility. Therefore, understanding the molecular mechanisms of TRAIL resistance and ways to sensitize these cells to undergo apoptosis by TRAIL are important issues for effective cancer therapy.

Selenium is an essential dietary nutrient for all mammalian species, which is primarily taken up from the soil by plants. Selenium exists in mostly organic forms in normal diets that is present in foods mainly in the forms of Se-Met, Se-Cys and Se-methylselenocysteine (CH3SeCys), whereas inorganic selenium either as selenite or selenate occurs much less frequently and in very low amounts (5). Several mechanisms have been proposed for the chemopreventive activity of selenium, including induction of apoptosis, inhibition of angiogenesis, and arrest of cell cycle (6-9). Although a number of selenocompounds are effective chemopreventive agents, Se-MSC continues to attract great attention because of its chemical nature, low toxicity, and significant chemopreventive effects (9).

The goal of this study is to evaluate Se-MSC as a sensitizer of TRAIL and to understand the mechanism of the synergy between Se-MSC and TRAIL against human renal cancer cells. In the present study, we found that Se-MSC treatment rendered human renal cancer cells more sensitive to the cytotoxic activity of TRAIL, suggesting that the combined treatment with Se-MSC and TRAIL may offer a safe and effective cancer therapy. We also found that Bcl-2 downregulation caused by $\mathrm{Se}-\mathrm{MSC}$ treatment contributes to the sensitizing effect of Se-MSC on TRAIL-induced apoptosis.

\section{Materials and methods}

Cells and materials. Caki cells were obtained from the American Type Culture Collection (ATCC: Rockville, MD). The culture medium used throughout these experiments was Dulbecco's modified Eagle's medium (DMEM), containing $10 \%$ fetal bovine serum (FBS), $20 \mathrm{mM}$ HEPES buffer and $100 \mu \mathrm{g} / \mathrm{ml}$ gentamicin. Se-MSC and TRAIL was directly added to cell cultures at the indicated concentrations. AntiBcl-2, anti-Bax, anti-Bcl-xL, anti-c-IAP1, anti-XIAP, antiprocaspase-3, anti-PARP, and anti-HSC70 antibodies were purchased from Santa Cruz Biotechnology Inc. (Santa Cruz, CA, USA). Soluble recombinant TRAIL was purchased from R\&D Systems (Minneapolis, MN). Se-MSC was obtained from Sigma Chemical Co. 
Western blotting. Cellular lysates were prepared by suspending $1 \times 10^{6}$ cells in $100 \mu 1$ of lysis buffer $(137 \mathrm{mM} \mathrm{NaCl}, 15 \mathrm{mM}$ EGTA, $0.1 \mathrm{mM}$ sodium orthovanadate, $15 \mathrm{mM} \mathrm{MgCl} 2,0.1 \%$ Triton X-100, $25 \mathrm{mM}$ MOPS, $100 \mu \mathrm{M}$ phenylmethylsulfonyl fluoride, and $20 \mu \mathrm{M}$ leupeptin, adjusted to $\mathrm{pH}$ 7.2). The cells were disrupted by sonication and extracted at $4^{\circ} \mathrm{C}$ for $30 \mathrm{~min}$. The proteins were electrotransferred to Immobilon-P membranes (Millipore Corp., Bedford, MA, USA). Detection of specific proteins was carried out with an ECL Western blotting kit according to the manufacturer's instructions.

Flow cytometry analysis. Approximately $1 \times 10^{6}$ Caki cells were suspended in $100 \mu \mathrm{l}$ of PBS, and $200 \mu 1$ of $95 \%$ ethanol were added while vortexing. The cells were incubated at $4{ }^{\circ} \mathrm{C}$ for $1 \mathrm{~h}$, washed with PBS, and resuspended in $250 \mu \mathrm{l}$ of $1.12 \%$ sodium citrate buffer $(\mathrm{pH} 8.4)$ together with $12.5 \mu \mathrm{g}$ of RNase. Incubation was continued at $37^{\circ} \mathrm{C}$ for $30 \mathrm{~min}$. The cellular DNA was then stained by applying $250 \mu 1$ of propidium iodide $(50 \mu \mathrm{g} / \mathrm{ml})$ for $30 \mathrm{~min}$ at room temperature. The stained cells were analyzed by fluorescent activated cell sorting (FACS) on a FACScan flow cytometer for relative DNA content based on red fluorescence.

Asp-Glu-Val-Asp-ase (DEVDase) activity assay. To evaluate DEVDase activity, cell lysates were prepared after their respective treatment with TRAIL or Se-MSC. Assays were performed in 96-well microtiter plates by incubating $20 \mu \mathrm{g}$ of cell lysates in $100 \mu \mathrm{l}$ of reaction buffer (1\% NP-40, $20 \mathrm{mM}$ Tris- $\mathrm{HCl}, \mathrm{pH} 7.5,137 \mathrm{mM} \mathrm{NaCl}, 10 \%$ glycerol) containing the caspases substrate [Asp-Glu-Val-Asp-chromophore-pnitroanilide (DVAD-pNA)] at $5 \mu \mathrm{M}$. Lysates were incubated at $37^{\circ} \mathrm{C}$ for $2 \mathrm{~h}$. Thereafter, the absorbance at $405 \mathrm{~nm}$ was measured with a spectrophotometer.

$R N A$ isolation and reverse transcriptase-polymerase chain reaction $(R T-P C R)$. Bcl-2 mRNA expression was determined by RT-PCR. Total cellular RNA was extracted from cells using the TRIzol reagent (Life Technologies). A cDNA was synthesized from $2 \mu \mathrm{g}$ of total RNA using M-MLV reverse transcriptase (Gibco-BRL, Gaithersburg, MD). The cDNA for Bcl-2 and actin were amplified by PCR with specific primers. The sequences of the sense and antisense primers for Bcl-2 were 5'-GGTGAACTGGGGGAGGATTGT-3' and 5'-CTTCAGAGACAGCCAGGAGAA, respectively. The sequence of the sense and antisense primers for actin were 5'-GGCATCGTCACCAACTGGGAC -3' and 5'-CGAT TTCCCGCTCGGCCGTGG-3', respectively. PCR products were analyzed by agarose gel electrophoresis and visualized by ethidium bromide.

Statistical analysis. Three or more separate experiments were performed. Statistical analysis was done by paired Student's t-test or ANOVA. A p $<0.05$ was considered to have pronounced difference between experimental and control groups.

\section{Results}

Se-MSC sensitizes TRAIL-mediated apoptosis in human renal cancer cells. To investigate the effect of Se-MSC on TRAIL-

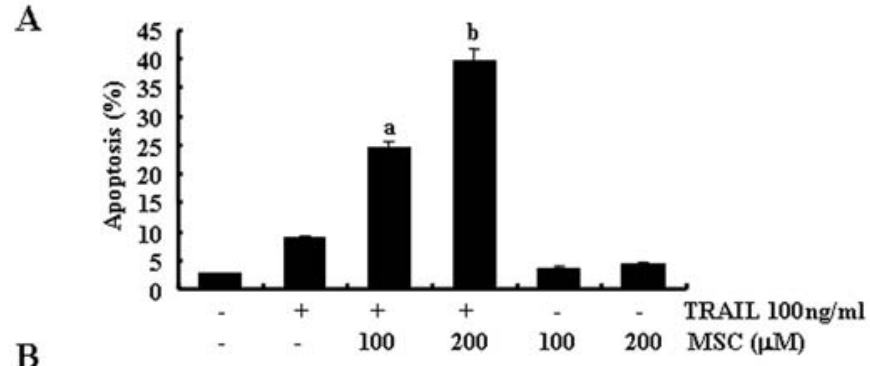

B

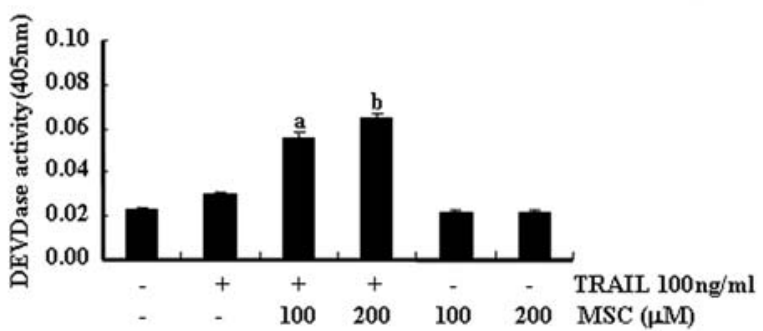

C

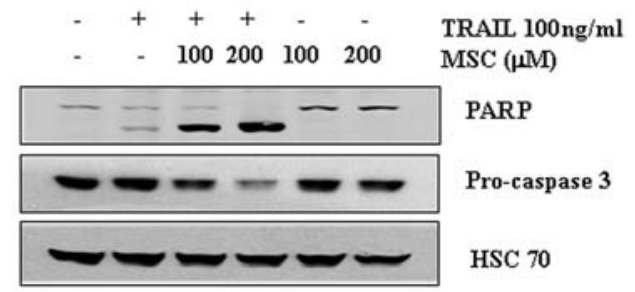

Figure 1. Se-MSC sensitizes cancer cells to TRAIL-induced apoptosis. (A) Caki cells were treated for $24 \mathrm{~h}$ with TRAIL $(100 \mathrm{ng} / \mathrm{ml})$ in either the absence or the presence of Se-MSC $(100$ or $200 \mu \mathrm{M})$. After $24 \mathrm{~h}$, apoptosis was analyzed as a sub-G1 fraction by FACS. a and $b, p<0.05$ for Se-MSC+TRAIL-treated cells versus control, Se-MSC-, or TRAIL-treated cells by ANOVA. (B) Activation of caspases in Se-MSC-sensitized TRAILinduced apoptosis. Cells were treated with the indicated concentrations of Se-MSC and TRAIL. Enzymatic activities of DEVDase were determined as described in Materials and methods. a and b, $\mathrm{p}<0.05$ for Se-MSC+TRAILtreated cells versus control, Se-MSC-, or TRAIL-treated cells by ANOVA. (C) Equal amounts of cell lysates $(40 \mu \mathrm{g})$ were subjected to electrophoresis and analyzed by Western blotting for PARP, procaspase-3, and HSC70 for normalization.

mediated apoptosis, human renal cancer Caki cells were treated with Se-MSC alone (10-200 $\mu \mathrm{M})$, TRAIL alone $(100 \mathrm{ng} / \mathrm{ml})$, or combination of Se-MSC and TRAIL. First, apoptosis in Caki cells was determined using flow cytometric analysis to detect hypodiploid cell populations. As shown in Fig. 1A, co-treatment of Caki cells with Se-MSC and TRAIL resulted in a markedly increased accumulation of sub-G1 phase cells, whereas treatment with Se-MSC alone or TRAIL alone did not. Second, we analyzed whether co-treatment with Se-MSC and TRAIL gave rise to the activation of caspase, a key executioner of apoptosis. Co-treatment of Caki cells with Se-MSC and TRAIL strongly stimulated DEVDase activity and led to a reduction of the protein levels of 32-kDa precursor together with a concomitant cleavage of PARP, a substrate protein of caspases (Fig. 1B and C). Taken together, these results indicate that treatment with Se-MSC sensitizes Caki cells to TRAIL-mediated apoptosis.

Se-MSC down-regulates Bcl-2 protein levels. To investigate the underlying mechanisms involved in Se-MSC plus TRAIL- 
A

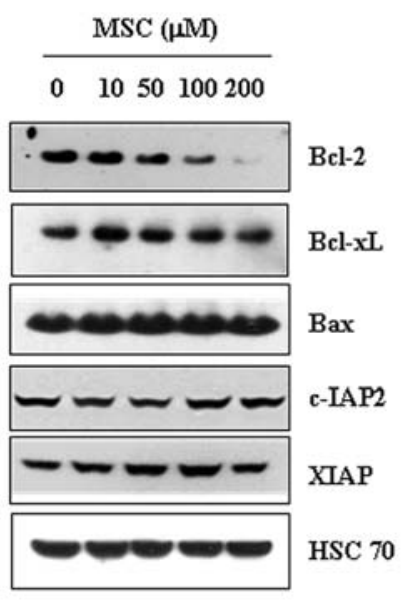

B

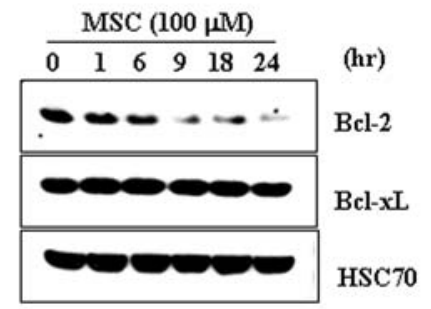

$\mathrm{C}$
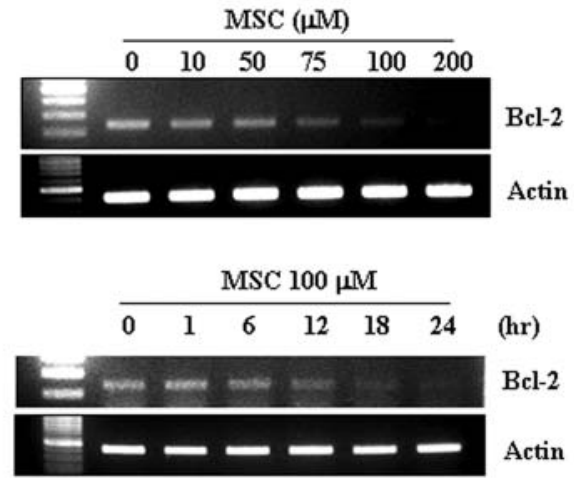

Figure 2. Se-MSC down-regulates Bcl-2 protein levels. (A) Caki cells were treated with the indicated concentrations of Se-MSC for $24 \mathrm{~h}$ and harvested in lysis buffer and equal amounts of cell lysates $(50 \mu \mathrm{g})$ were resolved by SDS-PAGE. Western blotting was performed using anti-Bcl-2, -Bcl-xL, -Bax, -XIAP, and -c-IAP2, or with anti-HSC70 antibody to serve as control for the loading of protein level. (B) Caki cells were treated with $100 \mu \mathrm{M}$ Sc-MSC for the indicated times and harvested in lysis buffer and equal amounts of cell lysates $(50 \mu \mathrm{g})$ were resolved by SDS-PAGE. Western blotting was performed using anti-Bcl-2 and -Bcl-xL, or with anti-HSC70 antibody to serve as control for the loading of protein level. (C) Caki cells were treated with the indicated concentrations of Se-MSC for $24 \mathrm{~h}$ (upper panel). Total RNA was isolated and RT-PCR analysis was performed as described in Materials and methods (upper panel). Caki cells were treated with $100 \mu \mathrm{M}$ Sc-MSC for the indicated times. Total RNA was isolated and RT-PCR analysis was performed as described in Materials and methods (lower panel). A representative study is shown; two additional experiments yielded similar results.

induced apoptosis, we analyzed the changes in the various apoptotic regulating proteins such as Bcl-2 family and inhibitor of apoptosis protein (IAP) family. As shown in Fig. 2A, combinatory treatment with Se-MSC and TRAIL led to reduction of the $\mathrm{Bcl}-2$ protein, but not $\mathrm{Bcl}-\mathrm{xL}$, Bax, c-IAP2 and XIAP, in a dose-dependent manner. Next, we carried out time kinetics studies to ascertain the down-regulation of Bcl-2 by Se-MSC in Caki cells. As shown in Fig. 2B, the protein levels of Bcl-2 were progressively degraded from 6 to $24 \mathrm{~h}$ after Se-MSC treatment. To further elucidate the mechanism responsible for the changes in amounts of Bcl-2 protein, we determined the levels of Bcl-2 mRNA by RT-PCR. Treatment with Se-MSC resulted in marked decrease of Bcl-2 mRNA levels, which were suppressed by Se-MSC in a dose- and time-dependent manner (Fig. 2C).

Sensitization to TRAIL-induced apoptosis by Se-MSC and TRAIL is dependent on caspases. To address the significance of caspase activation in Se-MSC plus TRAIL-mediated sensitization for TRAIL-induced apoptosis, we used a general and potent inhibitor of caspases, z-VAD-fmk (benzyloxycarbonyl-Val-Ala-Asp-fluoromethyl ketone). As shown in Fig. 3A, an increase in apoptotic population by the co-treatment with Se-MSC and TRAIL was significantly inhibited by pretreatment with $\mathrm{z}$-VAD-fmk. We also found that $\mathrm{z}$-VADfmk prevented all these caspase-related events such as cleavage of procasapse-3 and PARP and increase of DEVDase activity (Fig. 3B and C). These data clearly indicate that co-treatment with Se-MSC and TRAIL-induced apoptosis is associated with caspase activation.
The decreased expression of Bcl-2 might be involved in SeMSC plus TRAIL-induced apoptosis. To investigate whether the decreased expression of $\mathrm{Bcl}-2$ is important to induce apoptosis in Se-MSC plus TRAIL treated Caki cells, we first established Bcl-2 over-expressing cells. Excluding the possibility of clonal variation between the generated stable cell lines, pooled Caki/Vector and Caki/Bcl-2 cells were used in this study. Caki/Bcl-2 cells exhibited an increase in Bcl-2 expression compared with cells containing empty-vector only (Fig. 4A). Treatment with Se-MSC plus TRAIL for $24 \mathrm{~h}$ in Caki/Vector cells resulted in a markedly increased accumulation of sub-G1 phase cells. In contrast, the accumulation of sub-G1 phase induced by Se-MSC plus TRAIL was inhibited by Bcl-2 over-expression. As shown in Fig. 4B, protein levels of pro-caspase-3 decreased in Caki/Vector cells exposed to Se-MSC plus TRAIL for $24 \mathrm{~h}$, but its levels were rarely altered in Se-MSC plus TRAILtreated Caki/Bcl-2 cells. Subsequent Western blot analysis demonstrated that the proteolytic cleavage of PARP in Caki/Vector cells was more prominent than in Caki/Bcl-2 cells when exposed to Se-MSC plus TRAIL (Fig. 4B). Interestingly, the introduced $\mathrm{Bcl}-2$ protein was also slightly decreased by Se-MSC plus TRAIL treatment in Caki/Bcl-2 cells (Fig. 4B). Taken together, these results indicate that SeMSC plus TRAIL-induced down-regulation of Bcl-2 may be important for Se-MSC plus TRAIL-induced apoptosis.

Synergistic effects of combined treatment with TRAIL and BH3 mimetics. Since HA14-1 was originally identified as a functional inhibitor of the Bcl-2 (10), we tested whether 
A

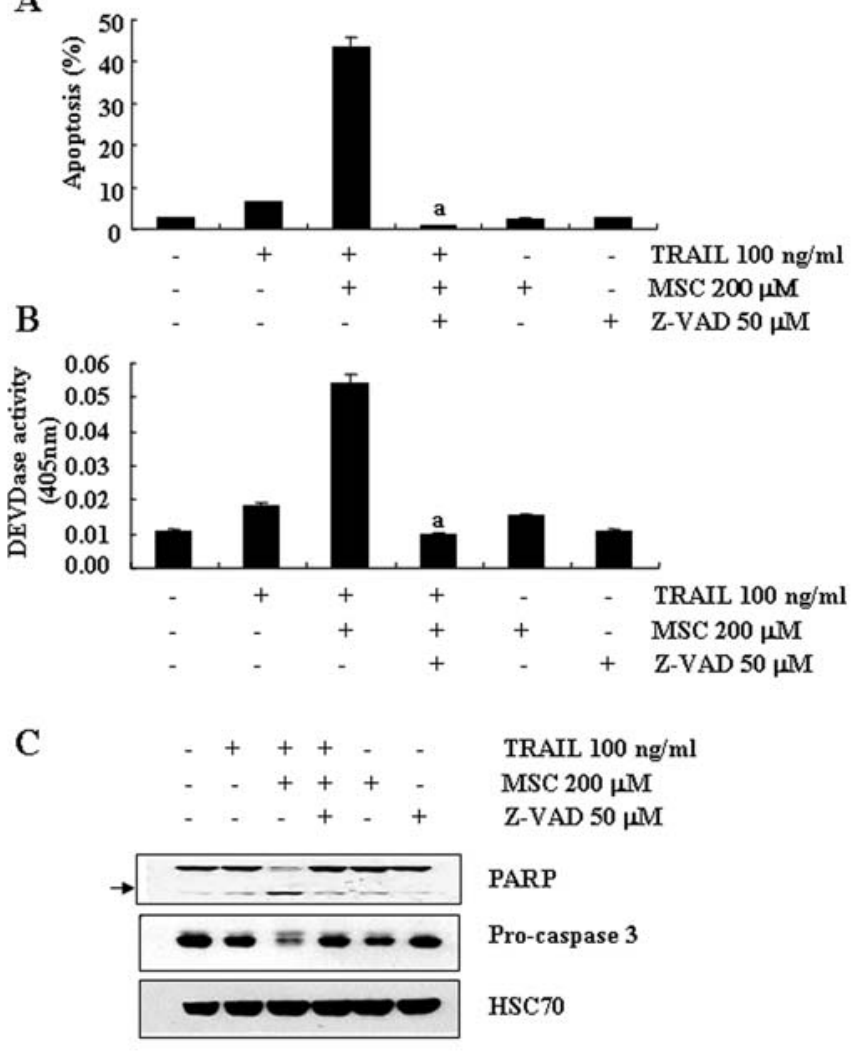

Figure 3. Se-MSC plus TRAIL treatment activated caspase-dependent pathways. (A) Effect of z-VAD-fmk on apoptosis induced by Se-MSC plus TRAIL. Caki cells were incubated with $50 \mu \mathrm{M}$ z-VAD-fmk or solvent for $1 \mathrm{~h}$ before treatment with Se-MSC $(200 \mu \mathrm{M})$ and/or TRAIL $(100 \mathrm{ng} / \mathrm{ml})$ for $24 \mathrm{~h}$. DNA contents of treated cells were evaluated after propidium iodide staining and apoptosis was measured as a sub-G1 fraction by FACS. Data are mean values obtained from three independent experiments and bars represent standard deviation. a, $\mathrm{p}<0.05$ compared to Se-MSC plus TRAIL-treated cells. (B) Effect of z-VAD-fmk on DEVDase activities in Se-MSC plus TRAIL treated cells. Cells were treated with the indicated concentrations of Se-MSC and TRAIL. Enzymatic activities of DEVDase were determined as described in Materials and methods. a, $\mathrm{p}<0.05$ compared to Se-MSC plus TRAIL-treated cells. (C) Equal amounts of cell lysates (40 $\mu \mathrm{g}$ ) were subjected to electrophoresis and analyzed by Western blotting for procaspase-3 and PARP. The proteolytic cleavage of PARP is indicated by an arrow. A representative study is shown; two additional experiments yielded similar results.

HA14-1 were capable of acting in a synergistic manner with TRAIL in Caki cells. As shown in Fig. 5A, co-treatment of Caki cells with HA14-1 and TRAIL resulted in a markedly increased accumulation of sub-G1 phase cells, whereas treatment with HA14-1 alone or TRAIL alone did not. Next, we analyzed whether co-treatment with HA14-1 and TRAIL gives rise to the activation of caspase- 3 as well as PARP cleavage. Co-treatment of Caki cells with HA14-1 and TRAIL strongly stimulated DEVDase activity and led to a reduction of the protein levels of 32-kDa precursor together with a concomitant cleavage of PARP, a substrate protein of caspases (Fig. 5B and C). Taken together, these results indicate that functional inhibition of the Bcl-2 by HA14-1 sensitizes Caki cells to TRAIL-mediated apoptosis.

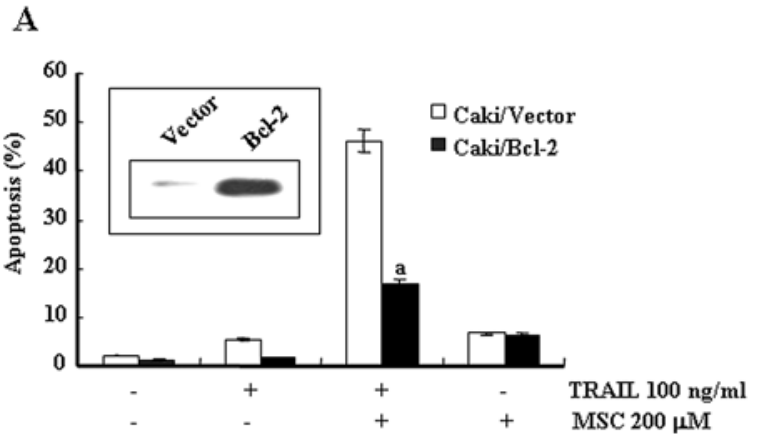

B

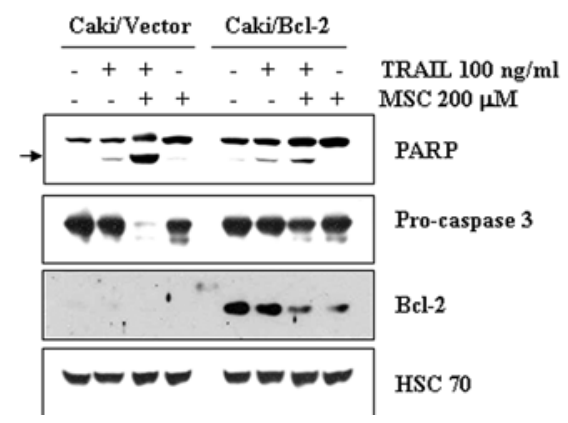

Figure 4. The decreased expression of Bcl-2 might be involved in Se-MSC plus TRAIL-induced apoptosis. (A) Caki/Vector and Caki/Bcl-2 cells were treated for $24 \mathrm{~h}$ with Se-MSC alone $(200 \mu \mathrm{M})$, TRAIL alone $(100 \mathrm{ng} / \mathrm{ml})$, or combination of Se-MSC and TRAIL. Apoptosis was analyzed as a sub-G1 fraction by FACS. Data shown are means \pm SD $(n=3)$. a, $p<0.05$ compared to Se-MSC plus TRAIL-treated Caki/Vector cells. (B) Equal amounts of cell lysates $(40 \mu \mathrm{g})$ were subjected to electrophoresis and analyzed by Western blotting for PARP, procaspase-3, Bcl-2, and HSC70. The proteolytic cleavage of PARP is indicated by an arrow.

\section{Discussion}

Selenium is currently being tested for cancer chemoprevention but only few studies have investigated its synergistic effects on TRAIL-mediated apoptosis in cancer cells. In general, the molecular mechanisms of selenium-mediated effects remain to be elucidated. In this report, we have presented evidence that selenium sensitized TRAIL-mediated apoptosis and down-regulated Bcl-2 expression in human renal cancer cells. Selenium-mediated Bcl-2 down-regulation is controlled at transcriptional levels at a dose- or time-dependent manner. Furthermore, we demonstrate that the synergistic effects of Se-MSC and TRAIL were resulted from the Bcl-2 downregulation induced by Se-MSC treatment, which was attenuated by the ectopic expression of $\mathrm{Bcl}-2$ expression vector. Pretreatment with Bcl-2 functional inhibitor, HA14-1 sensitizes Caki cells to TRAIL-mediated apoptosis.

Several studies demonstrate that TRAIL induces apoptosis in various human tumors (11-13), when investigated as a new cancer therapeutic agent. However, treatment with TRAIL alone may be insufficient for cancer therapy because some cancers display resistance to TRAIL. Therefore, natural compounds have gained considerable attention as cancer chemopreventive agents to escape TRAIL resistance in cancer cells or to sensitize cancer cells to TRAIL-induced apoptosis.

Recently, it has been generally accepted that $\mathrm{Bcl}-2$ protein blocks the release of cytochrome $\mathrm{c}$ from mitochondria to 
A

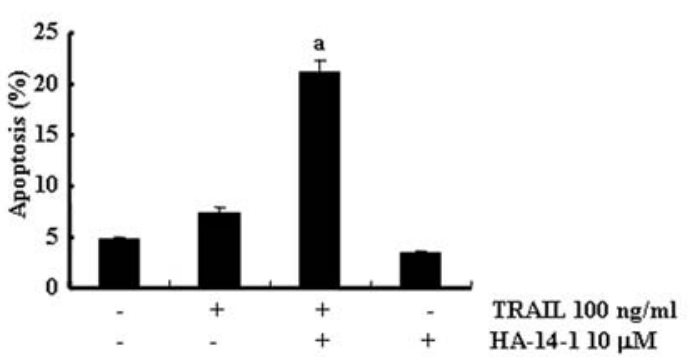

B
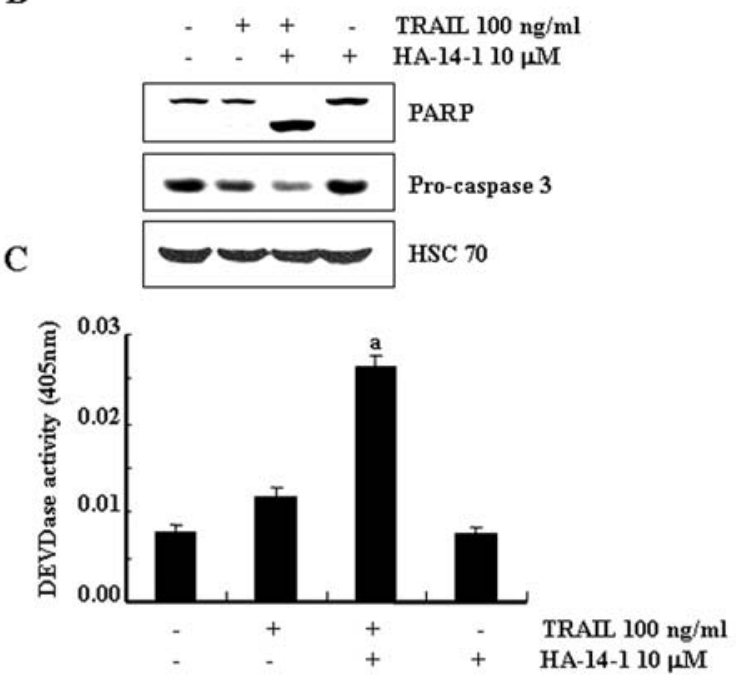

Figure 5. Synergistic effects of combined treatment with TRAIL and BH3 mimetics. (A) Caki cells were treated for $24 \mathrm{~h}$ with TRAIL $(100 \mathrm{ng} / \mathrm{ml})$ in either the absence or the presence of HA-12-1 (10 $\mu \mathrm{M})$. After $24 \mathrm{~h}$, apoptosis was analyzed as a sub-G1 fraction by FACS. a, p<0.05 for HA-12-1+TRAILtreated cells versus control, HA-12-1-, or TRAIL-treated cells by ANOVA. (B) Equal amounts of cell lysates $(40 \mu \mathrm{g})$ were subjected to electrophoresis and analyzed by Western blotting for PARP, procaspase-3, and HSC70 for normalization. (C) Activation of caspases in HA-12-1-sensitized TRAILinduced apoptosis. Cells were treated with the indicated concentrations of HA-12-1 and TRAIL. Enzymatic activities of DEVDase were determined as described in Materials and methods. a, $\mathrm{p}<0.05$ for HA-12-1+TRAIL-treated cells versus control, HA-12-1-, or TRAIL-treated cells by ANOVA.

the cytosol and therefore blocks the activation of caspases, which is pivotal for ensuing apoptotic process $(14,15)$. In addition, Bcl-2 itself is a pro-survival member of the family and its aberrant expression has been linked to a variety of different cancers, and cancer cells (16). Selenocystine (SeC) treatment suppressed the expression of pro-survival Bcl-2 family proteins, such as Bcl-2 and Bcl-xL, and moderately increased the expression levels of pro-apoptosis Bcl-2 family proteins, such as Bax and PUMA- $\alpha$ (17). There are several reports that chemopreventive agents such as sulforaphane, acetylsalicylic acid, and kaempferol inhibited Bcl-2 expression, increasing the sensitivity to TRAIL in cancer cells $(16,18,19)$. In our study, we found that Se-MSC treatment down-regulated $\mathrm{Bcl}-2$ expression at transcriptional level but failed to detect any differences in the levels of Bcl-xL and Bax proteins. It seems that Se-MSC, as an apoptogenic agent, might suppress the on-going expression of Bcl-2 in Caki cells, and thus triggers and maintains apoptosis during the time of TRAIL exposure. In addition, ectopic expression of Bcl-2 gene is inhibited in Se-MSC plus TRAIL-mediated apoptosis. These results indicate that Se-MSC-induced Bcl-2 down-regulation is critical to the sensitizing effect of
TRAIL-mediated apoptosis in Caki cells. Future studies will determine the mechanism of action of Se-MSC treatment for down-regulating $\mathrm{Bcl}-2$ at transcriptional levels.

Since Se-MSC increased the sensitivity to TRAIL via down-regulating Bcl-2 expression, we next determined whether the small molecule Bcl-2 inhibitor, HA14-1, could increase TRAIL sensitivity in human renal cancer cells. Recently, several studies showed that the synergism between HA14-1 treatment and TRAIL could be demonstrated in different cell lines and with different substances including TRAIL in renal carcinoma, glioma, and colon cancer cells (20-22). In our study, pretreatment with HA14-1 sensitized TRAIL-mediated apoptosis in renal cancer cells. This is in accordance with recent data indicating that co-treatment of renal carcinoma cell lines with HA14-1 further sensitized TRAIL-induced apoptosis (20).

Recent reports show that selenium-containing reagents, for example, methylseleninic acid (MSA) and inorganic selenium compounds (e.g., sodium selenite), sensitized prostate cancer cells to TRAIL-mediated apoptosis $(23,24)$. In addition, sodium selenite is reported to be specifically upregulated in DR5 expression, mediating selenium-mediated apoptosis in human prostate cancer cells (25). Although, sodium selenite can lead to Bax up-regulation and translocation into mitochondria sensitizing prostate cancer LNCaP cells to TRAIL (24), we failed to detect up-regulation of Bax and DR5 in Se-MSC-treated human renal cancer cells. Administration of TRAIL and MSA has been reported to produce synergistic effects on the induction of apoptosis in androgen-dependent LNCaP and androgen-independent DU-145 prostate cancer cells via down-regulation of cellular FLICE inhibitory protein (c-FLIP), a negative regulator of death receptor signaling as well as the activation of the mitochondrial pathway-mediated amplification loop (23). Yamaguchi et al, also showed that only MSA sensitized prostate cancer cells to TRAIL-mediated apoptosis among selenium-containing reagents, for example, MSA, sodium selenite, Se-Met and Se-MSC (23). In contrast, we observed that Se-MSC can sensitize human renal cancer cells to TRAIL-mediated apoptosis via down-regulation of Bcl-2 without any effect on c-FLIP expression (data not shown).

Collectively, the synergy between Se-MSC and TRAIL in human renal cancer cells is associated with decreased level of Bcl-2 and activation and caspase-dependent pathways. Thus, our present study suggests that selenium-based dietary compounds may help to overcome resistance to TRAILmediated apoptosis in renal cancer cells.

\section{Acknowledgements}

This work was supported by the Korea Science and Engineering Foundation through the MRC at Keimyung University (R13-2002-028-03001-0).

\section{References}

1. Srivastava RK: Intracellular mechanisms of TRAIL and its role in cancer therapy. Mol Cell Biol Res Commun 4: 67-75, 2000.

2. Ozoren N, Fisher MJ, Kim K, et al: Homozygous deletion of the death receptor DR4 gene in a nasopharyngeal cancer cell line is associated with TRAIL resistance. Int J Oncol 16: 917-925, 2000 . 
3. Wang S and El-Deiry WS: TRAIL and apoptosis induction by TNF-family death receptors. Oncogene 22: 8628-8633, 2003.

4. Ivanov VN, Bhoumik A and Ronai Z: Death receptors and melanoma resistance to apoptosis. Oncogene 22: 3152-3161, 2003.

5. Rikiishi H: Apoptotic cellular events for selenium compounds involved in cancer prevention. J Bioenerg Biomembr 39: 91-98, 2007.

6. Jiang C, Wang Z, Ganther $\mathrm{H}$ and $\mathrm{Lu} \mathrm{J}$ : Caspases as key executors of methyl selenium-induced apoptosis (anoikis) of DU-145 prostate cancer cells. Cancer Res 61: 3062-3070, 2001.

7. Wang Z, Jiang $\mathrm{C}$ and $\mathrm{Lu} \mathrm{J}$ : Induction of caspase-mediated apoptosis and cell-cycle G1 arrest by selenium metabolite methylselenol. Mol Carcinog 34: 113-120, 2002.

8. Lu J: Apoptosis and angiogenesis in cancer prevention by selenium. Adv Exp Med Biol 492: 131-145, 2001.

9. Ip C and Dong Y: Methylselenocysteine modulates proliferation and apoptosis biomarkers in premalignant lesions of the rat mammary gland. Anticancer Res 21: 863-867, 2001.

10. Chen J, Freeman A, Liu J, Dai Q and Lee RM: The apoptotic effect of HA14-1, a Bcl-2-interacting small molecular compound, requires Bax translocation and is enhanced by PK11195. Mol Cancer Ther 1: 961-967, 2002.

11. Abe K, Kurakin A, Mohseni-Maybodi M, Kay B and KhosraviFar R: The complexity of TNF-related apoptosis-inducing ligand. Ann NY Acad Sci 926: 52-63, 2000.

12. Griffith TS, Anderson RD, Davidson BL, Williams RD and Ratliff TL: Adenoviral-mediated transfer of the TNF-related apoptosis-inducing ligand/Apo-2 ligand gene induces tumor cell apoptosis. J Immunol 165: 2886-2894, 2000.

13. Ahmad M and Shi Y: TRAIL-induced apoptosis of thyroid cancer cells: potential for therapeutic intervention. Oncogene 19: 3363-3371, 2000.

14. Oliver L, Mahe B, Gree R, Vallette FM and Juin P: HA14-1, a small molecule inhibitor of Bcl-2, bypasses chemoresistance in leukaemia cells. Leuk Res 31: 859-863, 2007.

15. Otsuki Y: Apoptosis in human endometrium: apoptotic detection methods and signaling. Med Electron Microsc 34: 166-173, 2001.

16. Shankar S, Ganapathy S and Srivastava RK: Sulforaphane enhances the therapeutic potential of TRAIL in prostate cancer orthotopic model through regulation of apoptosis, metastasis, and angiogenesis. Clin Cancer Res 14: 6855-6866, 2008.
17. Chen T and Wong YS: Selenocystine induces caspaseindependent apoptosis in MCF-7 human breast carcinoma cells with involvement of $\mathrm{p} 53$ phosphorylation and reactive oxygen species generation. Int J Biochem Cell Biol 41: 666-676, 2009.

18. Kim KM, Song JJ, An JY, Kwon YT and Lee YJ: Pretreatment of acetylsalicylic acid promotes tumor necrosis factor-related apoptosis-inducing ligand-induced apoptosis by down-regulating BCL-2 gene expression. J Biol Chem 280: 41047-41056, 2005.

19. Siegelin MD, Reuss DE, Habel A, Herold-Mende C and von Deimling A: The flavonoid kaempferol sensitizes human glioma cells to TRAIL-mediated apoptosis by proteasomal degradation of survivin. Mol Cancer Ther 7: 3566-3574, 2008.

20. Heikaus S, van den Berg L, Kempf T, Mahotka C, Gabbert HE and Ramp U: HA14-1 is able to reconstitute the impaired mitochondrial pathway of apoptosis in renal cell carcinoma cell lines. Cell Oncol 30: 419-433, 2008

21. Hetschko H, Voss V, Horn S, Seifert V, Prehn JH and Kogel D: Pharmacological inhibition of Bcl-2 family members reactivates TRAIL-induced apoptosis in malignant glioma. J Neurooncol 86: 265-272, 2008

22. Sinicrope FA, Penington RC and Tang XM: Tumor necrosis factor-related apoptosis-inducing ligand-induced apoptosis is inhibited by Bcl-2 but restored by the small molecule Bcl-2 inhibitor, HA 14-1, in human colon cancer cells. Clin Cancer Res 10: 8284-8292, 2004.

23. Yamaguchi K, Uzzo RG, Pimkina J, et al: Methylseleninic acid sensitizes prostate cancer cells to TRAIL-mediated apoptosis. Oncogene 24: 5868-5877, 2005.

24. Hu H, Jiang C, Schuster T, Li GX, Daniel PT and Lu J: Inorganic selenium sensitizes prostate cancer cells to TRAILinduced apoptosis through superoxide/p53/Bax-mediated activation of mitochondrial pathway. Mol Cancer Ther 5: 1873-1882, 2006.

25. He Q, Rashid A, Rong R, Hillman MJ, Huang Y and Sheikh MS: Death receptor 5 regulation during selenium-mediated apoptosis in human prostate cancer cells. Cancer Biol Ther 1: 287-290, 2002. 